\title{
Mean value properties of solutions to the Helmholtz and modified Helmholtz equations
}

\author{
N. Kuznetsov \\ Laboratory for Mathematical Modelling of Wave Phenomena \\ Institute for Problems in Mechanical Engineering \\ Russian Academy of Sciences \\ V.O., Bol'shoy pr. 61, St. Petersburg, 199178 \\ Russian Federation \\ nikolay.g.kuznetsov@gmail.com
}

Mean value properties of solutions to the $m$-dimensional Helmholtz and modified Helmholtz equations are considered. An elementary derivation of these properties is given; it involves the Euler-Poisson-Darboux equation. Despite the similar form of these properties for both equations, their consequences distinguish essentially. The restricted mean value property for harmonic functions is amended so that a function, satisfying it in a bounded domain of a special class, solves the modified Helmholtz equation in this domain.

\section{Introduction}

In this note, we consider mean value properties of solutions to the $m$-dimensional Helmholtz and modified Helmholtz equations. These equations have the next level of complexity after the Laplace equation $\nabla^{2} u=0\left(\nabla=\left(\partial_{1}, \ldots, \partial_{m}\right)\right.$ is the gradient operator and $\left.\partial_{i}=\partial / \partial x_{i}\right)$. Indeed, the Helmholtz equation

$$
\nabla^{2} u+\lambda^{2} u=0, \quad \lambda \in \mathbb{R} \backslash\{0\},
$$

and the modified Helmholtz equation

$$
\nabla^{2} u-\mu^{2} u=0, \quad \mu \in \mathbb{R} \backslash\{0\}
$$

have the simplest possible additional terms which differ only by the sign of the coefficient at $u$. Solutions of these equations are assumed to be real; indeed, the results obtained for them can be extended to complex-valued ones by considering the real and imaginary part separately.

Since (1.1) turns into (1.2) by putting $\lambda=\mathrm{i} \mu$, it is natural to expect that mean value properties of solutions to these equations have similar form and this is really so. However, these properties are separated in some sense by those of harmonic functions; moreover, when we turn to their consequences such as various versions of maximum principle and Liouville's theorem the results differ essentially for solutions of (1.1) and (1.2). Like harmonic functions solutions of these equations are real analytic functions, but nonzero constants, which are harmonic, are not their solutions.

A few words about early studies of mean value properties of solutions to equations (1.1) and (1.2). It was Weber, who first derived the mean value formula for spheres for solutions to (1.1) in three dimensions; see his paper [1] published in 1868. In two dimensions, he obtained the analogous theorem next year; see [2]. However, the $m$-dimensional case was considered only in the classical book [3] (pp. 288 and 289) on the basis of the Pizzetti's mean value formula for polyharmonic functions [4]. To the best author's knowledge, no further studies of mean value 
properties for solutions to (1.1) had appeared since publication of the first edition of [3] in 1937 until 2021, when the mean value formula for balls and converse theorems for balls and spheres were obtained in [5].

Even less was published about mean value properties of solutions to equation (1.2). The mean value formula for spheres was derived by C. Neumann in the three-dimensional case; see his book [6], Chapter 9, $\S 3$, published in 1896. The $m$-dimensional formula for spheres appeared without proof in the Poritsky's article [7] published in 1938, but its results had been obtained ten years earlier being presented to the AMS in December 1928. Mistakingly, C. Neumann's formula is attributed to Weber in [7]. The mean value formula for balls and converse theorems for balls and spheres were formulated in [5] by analogy to those for solutions of the Helmholtz equation.

Since mean value properties of solutions to the Helmholtz equation were studied in detail in the recent paper [5], our attention is focussed primarily on the modified Helmholtz equation in the present note.

\section{New derivation of the mean value formulae}

By $B_{r}(x)=\{y:|y-x|<r\}$ we denote the open ball of radius $r$ centred at $x \in \mathbb{R}^{m}$; it is called admissible with respect to a domain $D \subset \mathbb{R}^{m}$ provided $\overline{B_{r}(x)} \subset D$, whereas $\partial B_{r}(x)$ is called admissible sphere in this case. Let $f$ be a function continuous on $D$, then

$$
M^{\circ}(f, x, r)=\frac{1}{\left|\partial B_{r}\right|} \int_{\partial B_{r}(x)} f(y) \mathrm{d} S_{y} \text { and } M^{\bullet}(f, x, r)=\frac{1}{\left|B_{r}\right|} \int_{B_{r}(x)} f(y) \mathrm{d} y
$$

are its mean values over admissible sphere and ball, respectively. Here $\mathrm{d} S$ is the surface area measure, $\left|\partial B_{r}\right|=m \omega_{m} r^{m-1}$ is the area of $\partial B_{r}$ and $\left|B_{r}\right|=\omega_{m} r^{m}$ is the volume of $B_{r}$; the volume of unit ball is $\omega_{m}=2 \pi^{m / 2} /[m \Gamma(m / 2)]$.

It is clear that these functions are continuous in $x$ and $r$; moreover, if $u \in C^{k}(D)$, then its mean values are in the same class in $x$ and $r$. By continuity we have that

$$
M^{\bullet}(f, x, 0)=M^{\circ}(f, x, 0)=f(x)
$$

whereas further identities for $M^{\circ}$ can be found in [8], Chapter IV.

2.1. The Euler-Poisson-Darboux equation. Let us show that the obvious relation

$$
m \int_{0}^{r} t^{m-1} M^{\circ}(u, x, t) \mathrm{d} t=r^{m} M^{\bullet}(u, x, r),
$$

where $u$ is a $C^{2}$-function, yields that

$$
M_{r r}^{\circ}+(m-1) r^{-1} M_{r}^{\circ}=\nabla_{x}^{2} M^{\circ} \text { for } r>0,
$$

which is referred to as the Euler-Poisson-Darboux equation.

Indeed, applying the Laplacian to both sides of (2.1), we obtain

$$
m \omega_{m} \int_{0}^{r} t^{m-1} \nabla_{x}^{2} M^{\circ}(u, x, t) \mathrm{d} t=\int_{B_{r}(0)} \nabla_{x}^{2} u(x+y) \mathrm{d} y .
$$


By Green's first formula the last integral is equal to

$$
\int_{|y|=r} \nabla_{x} u(x+y) \cdot \frac{y}{r} \mathrm{~d} S_{y}
$$

and changing variables this can be written as follows:

$$
r^{m-1} \frac{\partial}{\partial r} \int_{|y|=1} u(x+r y) \mathrm{d} S_{y}=m \omega_{m} r^{m-1} M_{r}^{\circ}(u, x, r) .
$$

Thus we arrive at the equality

$$
r^{m-1} M_{r}^{\circ}(u, x, r)=\int_{0}^{r} t^{m-1} \nabla_{x}^{2} M^{\circ}(u, x, t) \mathrm{d} t
$$

Differentiation of this relation with respect to $r$ yields (2.2).

2.2. Mean value formulae for spheres. To distinguish a solution of equation (1.1) from that of (1.2), we denote the latter by $v$ in what follows, keeping $u$ for a solution of (1.1). Thus, from now on we write equation (1.2) as follows:

$$
\nabla^{2} v-\mu^{2} v=0, \quad \mu \in \mathbb{R} \backslash\{0\} .
$$

As usual, $J_{\nu}$ and $I_{\nu}$ denote the Bessel function and the modified Bessel function of order $\nu$, respectively. Now, we are in a position to prove the following.

Theorem 2.1. Let $D$ be a domain in $\mathbb{R}^{m}$. If $u$ and $v \in C^{2}(D)$ solve equations (1.1) and (2.4), respectively, then the mean value equalities for spheres

$$
M^{\circ}(u, x, r)=a^{\circ}(\lambda r) u(x) \text { and } M^{\circ}(v, x, r)=\widetilde{a}^{\circ}(\mu r) v(x)
$$

hold for every admissible ball $B_{r}(x)$. Here

$$
a^{\circ}(\lambda r)=\Gamma\left(\frac{m}{2}\right) \frac{J_{(m-2) / 2}(\lambda r)}{(\lambda r / 2)^{(m-2) / 2}} \quad \text { and } \quad \widetilde{a}^{\circ}(\mu r)=\Gamma\left(\frac{m}{2}\right) \frac{I_{(m-2) / 2}(\mu r)}{(\mu r / 2)^{(m-2) / 2}},
$$

respectively.

Proof. We begin with a solution $v$ of equation (2.4) and then turn to the corresponding result for $u$ solving (1.1).

It is straightforward to show that $a(r)=\widetilde{a}^{\circ}(\mu r)$ is a unique solution the following Cauchy problem:

$$
a_{r r}+(m-1) r^{-1} a_{r}-\mu^{2} a=0, \quad a(0)=1, \quad a_{r}(0)=0 .
$$

This follows by virtue of the relations

$$
z I_{\nu+1}(z)+2 \nu I_{\nu}(z)-z I_{\nu-1}(z)=0, \quad\left[z^{-\nu} I_{\nu}(z)\right]^{\prime}=z^{-\nu} I_{\nu+1}(z)
$$

see [9], p. 79. In particular, the second one implies the second initial condition.

For the function $w(r, x)=\widetilde{a}^{\circ}(\mu r) v(x)-M^{\circ}(v, x, r)$, which is defined for all $x \in D$ and all $r \geqslant 0$ such that $\partial B_{r}(x)$ is admissible, we have

$$
w(x, 0)=0, \quad w_{r}(x, 0)=0 .
$$


The first initial condition is a consequence of the equalities $\widetilde{a}^{\circ}(0)=1$ and $M^{\circ}(v, x, 0)=v(x)$, whereas the second one follows in the limit as $r \rightarrow 0$ from equation (2.2) multiplied by $r$. Moreover, equations (2.2) and (2.7) yield that

$$
w_{r r}+(m-1) r^{-1} w_{r}-\mu^{2} w=0 \text { for } r>0 .
$$

Since the latter Cauchy problem has only a trivial solution, we obtain that the second equality (2.5) holds with the coefficient given by the second formula (2.6).

To obtain the first equality (2.5) one has to repeat these considerations with $a(r)=a^{\circ}(\lambda r)$ and $w(r, x)=a^{\circ}(\lambda r) u(x)-M^{\circ}(u, x, r)$; moreover, the relations

$$
z J_{\nu+1}(z)-2 \nu J_{\nu}(z)+z J_{\nu-1}(z)=0, \quad\left[z^{-\nu} J_{\nu}(z)\right]^{\prime}=-z^{-\nu} J_{\nu+1}(z)
$$

must be used instead of (2.8); see [9], pp. 45 and 66, where these formulae are derived.

For $m=3$ the mean value formulae for spheres have particularly simple form. Indeed,

$$
a^{\circ}(\lambda r)=\frac{\sin \lambda r}{\lambda r} \text { and } \widetilde{a}^{\circ}(\mu r)=\frac{\sinh \mu r}{\mu r},
$$

because $J_{1 / 2}(z)=\sqrt{2 /(\pi z)} \sin z$ and $I_{1 / 2}(z)=\sqrt{2 /(\pi z)} \sinh z$. For arbitrary $m$, we see that $a^{\circ}(\lambda r)$ and $\widetilde{a}^{\circ}(\mu r) \rightarrow 1$ as $\lambda, \mu \rightarrow 0$, and so both equalities (2.5) recover in the limit the mean value theorem for spheres valid for harmonic functions. However, the second formula (2.8) shows that $\widetilde{a}^{\circ}(\mu r)$ approaches unity from above, whereas $a^{\circ}(\lambda r)$ does this from below according to the analogous differentiation formula for $J_{\nu}$.

2.3. Mean value formulae for balls. An immediate consequence of (2.5) and (2.6) are mean value properties for balls.

Corollary 2.1. Let $D$ be a domain in $\mathbb{R}^{m}$. If $u$ and $v \in C^{2}(D)$ solve equations (1.1) and (2.4), respectively, then the mean value equalities for balls

$$
M^{\bullet}(u, x, r)=a^{\bullet}(\lambda r) u(x) \text { and } M^{\bullet}(v, x, r)=\widetilde{a}^{\bullet}(\mu r) v(x)
$$

hold for every admissible ball $B_{r}(x)$. Here

$$
a^{\bullet}(\lambda r)=\Gamma\left(\frac{m}{2}+1\right) \frac{J_{m / 2}(\lambda r)}{(\lambda r / 2)^{m / 2}} \quad \text { and } \quad \widetilde{a}^{\bullet}(\mu r)=\Gamma\left(\frac{m}{2}+1\right) \frac{I_{m / 2}(\mu r)}{(\mu r / 2)^{m / 2}},
$$

respectively.

Proof. To obtain the first formula (2.10) with the coefficient given in (2.11) it suffices to write the first formula (2.5) in the form

$$
m \omega_{m} a^{\circ}(\lambda \rho) u(x)=\int_{\partial B_{1}(0)} u(x+\rho y) \mathrm{d} S_{y}
$$

multiply by $\rho^{m-1}$, and integrate with respect to $\rho$ within an admissible ball. To this end formula 1.8.1.21, [10]:

$$
\int_{0}^{x} x^{1+\nu} J_{\nu}(x) \mathrm{d} x=x^{1+\nu} J_{\nu+1}(x), \Re \nu>-1 .
$$


is helpful. Applying it with $\nu=(m-2) / 2$ while integrating the first expression (2.6), the required result follows.

For obtaining the second formula (2.10) with the coefficient given in (2.11) one has to use the same procedure, but applying formula (2.12) with Bessel functions changed to modified ones; see 1.11.1.5 in [10].

As in the case of spheres, $a^{\bullet}(\lambda r)$ and $\widetilde{a}^{\bullet}(\mu r)$ tend to unity as $\lambda, \mu \rightarrow 0$ (from below and above, respectively), thus recovering in the limit the mean value theorem for balls valid for harmonic functions.

\section{Applications of mean value properties}

First we notice an interesting feature of the function $a^{\circ}(\lambda r)$ for $m=2$. In this case, the sequence $\left\{J_{0}\left(j_{0, n}|x| / r\right)\right\}_{n=1}^{\infty}$ (as usual, $j_{\nu, n}$ denotes the $n$th positive zero of $J_{\nu}$ ) consists of eigenfunctions satisfying equation (1.1) in $B_{r}(0)$ and the Dirichlet boundary condition on $\partial B_{r}(0)$ provided $\lambda^{2}$ is determined from the relation $\lambda r=j_{0, n}$. Since $J_{0}(0)=1$, the validity of the mean value formula for $\partial B_{r}(0)$ for each of these eigenfunctions is guaranteed by the equality $a^{\circ}\left(j_{0, n}\right)=0$. On the other hand, all other Dirichlet eigenfunctions vanish at the origin, and so the mean value formula holds for them irrespective of the value attained by $a^{\circ}$.

Similarly, the sequence $\left\{J_{0}\left(j_{1, n}|x| / r\right)\right\}_{n=1}^{\infty}$ consists of eigenfunctions satisfying equation (1.1) in $B_{r}(0)$ and the Neumann boundary condition on $\partial B_{r}(0)$ provided $\lambda^{2}$ is determined from the relation $\lambda r=j_{1, n}$; notice that $J_{0}^{\prime}(z)=-J_{1}(z)$. Each of these eigenfunctions has the zero mean value for $B_{r}(0)$, being orthogonal to a nonzero constant - the Neumann eigenfunction corresponding to $\lambda^{2}=0$. Despite the equality $J_{0}(0)=1$, the mean value formula for $B_{r}(0)$ holds for each of these eigenfunctions; this time because $a^{\bullet}\left(j_{1, n}\right)=0$. As above, all Neumann eigenfunctions without central symmetry vanish at the origin, and so the mean value formula for $B_{r}(0)$ holds for them irrespective of the value attained by $a^{\bullet}$.

In the remaining part of this section, the results obtained in $\S 2$ are used for proving several properties of solutions to equations (1.1) and (2.4).

The second formula (2.9) implies that $a^{\circ}$ decreases monotonically on the interval $\left(0, j_{m / 2,1}\right)$; also, it is positive on the smaller interval $\left(0, j_{(m-2) / 2,1}\right)$ and changes sign at $\lambda r=j_{(m-2) / 2,1}$ (it is clear that the function $a^{\bullet}$ has similar properties). Combining the latter fact and the first equality (2.5), we arrive at the following.

Proposition 3.1. Let $D$ be a domain in $\mathbb{R}^{m}$, and let $u \in C^{2}(D)$ do not vanish identically and satisfy equation (1.1) in $D$ with $\lambda>0$. If for some $x \in D$ there exists $B_{r_{*}}(x) \subset D$ with $r_{*}>j_{(m-2) / 2,1} / \lambda$, then $u$ vanishes somewhere in $B_{r_{*}}(x)$. Moreover, the nodal set

$$
N(u)=\{y \in D: u(y)=0\}
$$

is an $m-1$-dimensional hypersurface in $D$.

Proof. If $u(x)=0$, then a zero already exists and we denote it by $x_{0}$. Let $u(x)>0$, and let $\delta>0$ be sufficiently small and such that $r_{+}=\left(j_{(m-2) / 2,1}+\delta\right) / \lambda<r_{*}$. Then $M^{\circ}\left(u, x, r_{+}\right)<0$ because $a^{\circ}\left(\lambda r_{+}\right)<0$ in view of the behaviour of $J_{(m-2) / 2}(\lambda r)$. Therefore, $u$ attains a negative value at some point on $\partial B_{r_{+}}(x) \subset B_{r_{*}}(x)$. By continuity $u$ vanishes at some point $x_{0} \in B_{r_{*}}(x)$ lying between $x$ and the obtained point on $\partial B_{r_{*}}(x)$. 
If $u(x)<0$, then applying the same considerations to $-u$ we obtain the required point $x_{0}$ such that $-u\left(x_{0}\right)=0$.

Thus in all possible cases, there exists $x_{0} \in B_{r_{*}}(x)$ such that $u\left(x_{0}\right)=0$, and so $M^{\bullet}\left(u, x_{0}, r\right)$ vanishes provided $\overline{B_{r}\left(x_{0}\right)} \subset D$. This implies that $B_{r}\left(x_{0}\right)$ is divided by an analytic $m-1$ dimensional hypersurface into parts, where $u>0$ and $u<0$; indeed, $u$ is a real analytic function in $D$ not vanishing identically there. By analyticity this hypersurface extends from $B_{r}\left(x_{0}\right)$ to $D$.

To illustrate Proposition 3.1, let us consider the function $u_{+}(x)=|x|^{-1} \sin \lambda|x|$. It satisfies equation (1.1) in $\mathbb{R}^{3}$, and $N\left(u_{+}\right)=\cup_{k=1}^{\infty} S_{k}$-the union of spheres $S_{k}=\partial B_{\pi k / \lambda}(0)$ - is its nodal set. Being positive in $B_{\pi / \lambda}(0)$, this function demonstrates that the restriction $r_{*}>j_{(m-2) / 2,1} / \lambda$ $\left(j_{(m-2) / 2,1} / \lambda=\pi / \lambda\right.$ in this case $)$ is essential. Moreover, $u_{+}$easily provides various domains enclosing balls of the radius $r_{*}>\pi / \lambda$, and so containing parts of $S_{k}$ or the whole ones as its nodal sets in these domains. For example, the domain $B_{(\pi+\epsilon) / \lambda}(0)$, where $\epsilon>0$ is sufficiently small, has the whole $S_{1}$ as the closed $N\left(u_{+}\right)$. On the other hand, $N\left(u_{+}\right)$consists of two pieces within $B_{(\pi+\epsilon) / \lambda}((3 \pi / 2,0,0))$ ( $\epsilon$ is the same as above): one lying on $S_{1}$ and the other one on $S_{2}$. Of course, there is a plethora of more complicated examples.

We recall that mean value properties of harmonic functions have two important consequences: the strong maximum principle and Liouville's theorem. The first asserts that a function harmonic in a domain $D$ cannot have local maxima or minima in $D$; moreover, if it is continuous in $\bar{D}$, which is bounded, then its maximum and minimum are attained on $\partial D$; see [11], Chapter 11, $\S 8$. The second theorem says that every harmonic function bounded below (or above) on $\mathbb{R}^{m}$ is a constant; see [11], Chapter 12, $\S 4$. Let us consider whether mean value properties of solutions to equations (1.1) and (2.4) imply analogous theorems.

It is clear that the function $u_{+}$violates both these assertions, and so they do not hold for solutions of equation (1.1). How this is related to the inequalities $a^{\circ}(\lambda r)<1$ and $a^{\bullet}(\lambda r)<1$ for $\lambda r>0$ is an open question.

Another question is about analogous theorems for solutions of equation (2.4). They are also not true as formulated above; indeed, the function $u_{-}(x)=|x|^{-1} \sinh |x|$ satisfies this equation on $\mathbb{R}^{3}$ (with $\mu=1$ ), and has the local (and global) minimum equal to one at the origin. Moreover, $u_{-}$violates the generalized Liouville theorem (see [12], p. 198), which guarantees that a harmonic function is constant on $\mathbb{R}^{m}$ under a weaker assumption than in Liouville's theorem.

Thus some extra restrictions must be imposed in order to convert formulations of the maximum principle and Liouville's theorem into true ones for solutions of equation (2.4). It is easy to obtain them for the whole $\mathbb{R}^{m}$, in which case, in view of self-similarity, it is sufficient to restrict ourselves to the equation:

$$
\nabla^{2} v-v=0 \text { in } \mathbb{R}^{m}
$$

indeed, it follows from (2.4) by the proper change of variables.

Let us consider the asymptotic behaviour of the function $\widetilde{a}^{\circ}(r)$ as $r \rightarrow \infty$. The formula

$$
I_{\nu}(z)=\frac{\mathrm{e}^{z}}{\sqrt{2 \pi z}}\left[1+O\left(|z|^{-1}\right)\right], \quad|\arg z|<\pi / 2
$$

whose principal term does not depend on $\nu$, is valid as $|z| \rightarrow \infty$; see [9], p. 80. Combining (3.2) 
and the second formula (2.6), one obtains

$$
\widetilde{a}^{\circ}(r)=\frac{\Gamma(m / 2) 2^{(m-3) / 2}}{\sqrt{\pi}} \frac{\mathrm{e}^{r}}{r^{(m-1) / 2}}\left[1+O\left(r^{-1}\right)\right] \quad \text { as } r \rightarrow \infty .
$$

Now we are in a position to prove the following version of Liouville's theorem.

Theorem 3.1. Let $v$ be a solution of (3.1) on $\mathbb{R}^{m}$. If the inequality

$$
|v(x)| \leqslant C(1+|x|)^{n} \text { holds for all } x \in \mathbb{R}^{m}
$$

with some $C>0$ and a nonnegative integer $n$, then $v$ vanishes identically.

Proof. Let us write the second formula (2.5) in the form

$$
m \omega_{m} \widetilde{a}^{\circ}(r) v(x)=\int_{\partial B_{1}(0)} v(x+r y) \mathrm{d} S_{y} .
$$

Then (3.3) and the asymptotic formula for $\widetilde{a}^{\circ}(r)$ imply the inequality

$$
|v(x)| \leqslant \widetilde{C}(1+|x|+r)^{n} \frac{r^{(m-1) / 2}}{\mathrm{e}^{r}} \text { for all } x \in \mathbb{R}^{m} \text { and all } r>0
$$

with some $\widetilde{C}>0$. Letting $r \rightarrow \infty$, the required assertion follows.

Inequality (3.3) implies that a solution to equation (3.1) is trivial regardless that $n>0$ can be taken arbitrarily large. On the other hand, if the same inequality is imposed on a harmonic function, then it is a (harmonic) polynomial, whose degree is less than or equal to $n$; see [13], p. 290.

The next assertion concerns the behaviour of $|v|$ for a nontrivial solution $v$ to equation (2.4).

Proposition 3.2. Let $D$ be a domain in $\mathbb{R}^{m}$, and let a nonvanishing identically function $v \in C^{2}(D)$ satisfy equation (2.4) there. Then for every $x \in D$ there exists $y \in D$ such that $|v(y)|>|v(x)|$.

Proof. Without loss of generality, we assume that $v(x) \geqslant 0$; indeed, $-v$ ought to be considered instead of $v$ otherwise. Then we have that $M^{\circ}(v, x, r) \geqslant 0$ for an admissible sphere $\partial B_{r}(x)$ because $\widetilde{a}^{\circ}(\mu r)>1$; moreover, $v(x)<M^{\circ}(v, x, r)$ by Theorem 2.1. The last inequality implies that there exists $y \in \partial B_{r}(x) \subset D$ such that $v(y)>v(x)$, which completes the proof.

An immediate consequence of this proposition is the weak maximum principle for solutions to equation (2.4); see [14, $\S 3.1$, for the approach applicable to general elliptic equations.

Theorem 3.2. Let $D$ be a bounded domain in $\mathbb{R}^{m}$. If $v \in C^{2}(D) \cap C^{0}(\bar{D})$ satisfies equation (2.4) in $D$, then

$$
\sup _{x \in D}|v(x)|=\max _{x \in \partial D}|v(x)|
$$

Proof. In the case of nonvanishing identically function $v$, we take a sequence $\left\{x_{k}\right\}_{k=1}^{\infty} \subset D$ such that $\left|v\left(x_{k}\right)\right| \rightarrow \sup _{x \in D}|v(x)|$ as $k \rightarrow \infty$. Since $D$ is bounded, $\left\{x_{k}\right\}_{k=1}^{\infty}$ has a limit point in $\bar{D}$, say $x_{0}$, and $\left|v\left(x_{0}\right)\right|=\sup _{x \in D}|v(x)|$ by continuity. Moreover, $x_{0} \in \partial D$; indeed, if $x_{0} \in D$, then Proposition 3.2 implies that there exists $y \in D$ such that $|v(y)|>\left|v\left(x_{0}\right)\right|$, which is impossible. Now (3.4) follows from the equality $\sup _{x \in D}|v(x)|=\max _{x \in \bar{D}}|v(x)|$ valid for $v \in C^{0}(\bar{D})$. 
An immediate consequence of this theorem (see [6], p. 260, for the original formulation) is the uniqueness of a solution to the Dirichlet problem for equation (2.4) in a bounded domain as well as the continuous dependence of solutions to this problem on boundary data.

\section{A converse of Theorem 2.1 concerning equation (2.4)}

In the recent paper [5], it is shown that if the first (second) equality (2.5) is valid for every $x \in D$ and for all $r \in(0, r(x))$, where $B_{r(x)}(x)$ is admissible, then $u$ ( $v$, respectively) is a solution of the Helmholtz (modified Helmholtz, respectively) equation. Thus, mean value properties characterize solutions of these equations in the same way as the Koebe theorem characterizes harmonic functions; see the classical Kellogg's book [15], pp. 226, 227. The next step was made by Kellogg; his paper [16] of 1934 initiated a long series of publications dealing with the so-called restricted mean value properties in order to characterize harmonicity. Baxter generalized Kellogg's result in his note [17] and introduced the following definition of the restricted properties.

Definition 4.1. A real-valued function $f$ defined on an open $G \subset \mathbb{R}^{m}$ is said to have the restricted mean value property with respect to balls (spheres) if for each $x \in G$ there exists a single ball (sphere) centred at $x$ of radius $r(x)$ such that $B_{r(x)}(x) \subset G$ and the average of $f$ over this ball (its boundary) is equal to $f(x)$.

Some applications of these properties and their further generalizations used in the theory of harmonic functions were outlined in the survey paper [18], § 3. In the present note, Definition 4.1 is amended in order to accommodate it for characterizing solutions of the modified Helmholtz equation.

Definition 4.2. A real-valued function $f$ defined on an open $G \subset \mathbb{R}^{m}$ is said to have the modified restricted mean value property with respect to spheres if for each $x \in G$ there exists a single sphere centred at $x$ of radius $r(x)$ such that $B_{r(x)}(x) \subset G$ and the second equality (2.5) with $r=r(x)$ holds for $f$.

Theorem 4.1. Let $D \subset \mathbb{R}^{m}$ be a bounded domain such that the Dirichlet problem for the modified Helmholtz equation is soluble in $C^{2}(D) \cap C^{0}(\bar{D})$ for every continuous function given on $\partial D$. If $v \in C^{0}(\bar{D})$ has the modified restricted mean value property in $D$ with respect to spheres, then $v$ satisfies equation (2.4) in $D$.

Proof. First, let us show that the theorem's assumptions yield that

$$
\max _{x \in \bar{D}}|v(x)|=\max _{x \in \partial D}|v(x)| .
$$

Reasoning by analogy with the proof of Proposition 3.2, we see that the modified restricted mean value property implies that for every $x \in D$ there exists $y \in D$ such that $|v(y)|>|v(x)|$. Then the considerations used in the proof of Theorem 3.2 yield (4.1).

Let $f$ denote the trace of $v$ on $\partial D$; then there exists $v_{0} \in C^{0}(\bar{D})$ solving the Dirichlet problem for equation (2.4) in $D$ with $f$ as the boundary data. Hence $v_{0}$ satisfies the second equality (2.5) for all $x \in D$ and all admissible $\partial B_{r}(x)$, and so the modified restricted mean value property is valid for $v-v_{0}$. Then the weak maximum principle (4.1) holds for $v-v_{0}$, thus implying that $v \equiv v_{0}$ in $D$ because $v \equiv v_{0}$ on $\partial D$. Then $v$ also satisfies the second equality (2.5) for all $x \in D$ and all admissible $\partial B_{r}(x)$. Now, Theorem 7 formulated in [5] yields that $v$ is a solution of equation (2.4) in $D$. 
Since the proof of the latter theorem is omitted in [5], we include it in this note for the sake of completeness.

Theorem 4.2. Let $D$ be a bounded domain in $\mathbb{R}^{m}$. If a real-valued $v \in C^{0}(D)$ satisfies the second equality (2.5) with some $\mu>0$ for every $x \in D$ and all $r \in(0, r(x))$, where the ball $B_{r(x)}(x)$ is admissible, then $v$ is a solution of equation (2.4) in $D$.

Proof. First, let is necessary to show that $v$ is smooth and for this purpose we use the method applied by Mikhlin in his proof of the corresponding theorem for harmonic functions; see [1], Chapter $11, \S 7$.

Let $D^{\prime}$ be a subdomain of $D$ whose closure $\overline{D^{\prime}} \subset D$ is separated from $\partial D$ by a layer formed by parts of balls located within $D$; each of these balls has its centre on $\partial D$ and radius $2 \epsilon$. By $\omega_{\epsilon}(|y-x|)=\omega_{\epsilon}(r)$ we denote the mollifier considered in [11], Chapter $1, \S 1$. Let $x \in D^{\prime}$, then, multiplying the second equality (2.5) by $\omega_{\epsilon}(r)$, we obtain

$$
v(x) \widetilde{a}^{\circ}(\mu r)\left|\partial B_{r}\right| \omega_{\epsilon}(r)=\omega_{\epsilon}(r) \int_{\partial B_{r}(x)} v(y) \mathrm{d} S_{y},
$$

where $\widetilde{a}^{\circ}$ is defined by (2.6) . Integration with respect to $r$ over $(0, \epsilon)$ yields

$$
v(x) c(\mu, \epsilon)=\int_{B_{\epsilon}(x)} v(y) \omega_{\epsilon}(|y-x|) \mathrm{d} y=\int_{D} v(y) \omega_{\epsilon}(|y-x|) \mathrm{d} y .
$$

Here the last equality follows from the fact that $x \in D^{\prime}$, whereas $\omega_{\epsilon}(|y-x|)$ vanishes outside $B_{\epsilon}(x)$. Moreover,

$$
c(\mu, \epsilon)=\int_{0}^{\epsilon} \widetilde{a}^{\circ}(\mu r)\left|\partial B_{r}\right| \omega_{\epsilon}(r) \mathrm{d} r>0,
$$

because $\widetilde{a}^{\circ}(\mu r)>1$. Since $\omega_{\epsilon}$ is infinitely differentiable, the obtained representation shows that $v \in C^{\infty}\left(D^{\prime}\right)$. By taking $\epsilon$ arbitrarily small, we see that $v \in C^{\infty}(D)$.

Now we are in a position to show that $v$ is a solution of equation (2.4) in $D$. Let $x \in D$ and let $r(x)>0$ be such that $B_{r(x)}(x)$ is admissible. Since the second equality (2.5) holds for all $r \in(0, r(x))$, for any such $r$ the second equality (2.10) holds as well (it follows from (2.5) by integration). Applying the Laplacian to the integral on the left-hand side of the second equality (2.10), we obtain

$$
\int_{|y|<r} \nabla_{x}^{2} v(x+y) \mathrm{d} y=\int_{|y|=r} \nabla_{x} v(x+y) \cdot \frac{y}{r} \mathrm{~d} S_{y} .
$$

Here the equality is a consequence of Green's first formula. By changing variables this can be written as follows:

$$
r^{m-1} \frac{\partial}{\partial r} \int_{|y|=1} v(x+r y) \mathrm{d} S_{y}=\left|\partial B_{1}(0)\right| r^{m-1} \frac{\partial}{\partial r} M^{\circ}(v, x, r),
$$

where $M^{\circ}(v, x, r)=\widetilde{a}^{\circ}(\mu r) v(x)$. In view of the second equality (2.8), we have that

$$
\frac{\partial}{\partial r} M^{\circ}(v, x, r)=-\frac{\mu I_{m / 2}(\mu r)}{(\mu r / 2)^{(m-2) / 2}} v(x) \text {. }
$$

Combining the above considerations and the second equality (2.10), we conclude that for every $x \in D$ the equality

$$
\int_{|y|<r}\left[\nabla_{x}^{2} v-\mu^{2} v\right](x+y) \mathrm{d} y=0 \text { holds for all } r \in(0, r(x)) .
$$


Thus, in each $B_{r}(x)$ there exists $y(r, x)$ such that $\left[\nabla^{2} v-\mu^{2} v\right](y(r, x))=0$. Since $y(r, x) \rightarrow x$ as $r \rightarrow 0$, we obtain by continuity that $v$ satisfies the modified Helmholtz equation at every $x \in D$.

The question about domains in which the Dirichlet problem for an elliptic equation is soluble has a long history which goes back to George Green's Essay on the Application of Mathematical Analysis to the Theories of Electricity and Magnetism published in 1828. This problem for the Laplace equation was posed in it for the first time. The final answer when the Dirichlet problem for harmonic functions has a solution was given by Wiener [19] in 1924; the notion of capacity was introduced for this purpose.

The class of domains such that the Dirichlet problem is soluble is the same for the modified Helmholtz equation and for the Laplace equation. This follows from the results of Oleinik [20] and Tautz [21]; they demonstrated independently and published in 1949 that this fact about the solubility of the Dirichlet problem is a common characteristic which is true for elliptic equations of rather general form (see the monograph [22], Chapter IV, $\S 28$, for a review of related papers).

\section{References}

1. H. Weber, "Ueber einige bestimmte Integrale," J. reine angew. Math. 69, 222-237 (1868).

2. H. Weber, "Ueber die Integration der partiellen Differentialgleichung: $\frac{\partial^{2} u}{\partial x^{2}}+\frac{\partial^{2} u}{\partial y^{2}}+k^{2} u=0$," Math. Ann. 1, 1-36 (1869).

3. R. Courant and D. Hilbert, Methods of Mathematical Physics, vol. 2, Partial Differential Equations, Wiley-Interscience, New York (1962).

4. P. Pizzetti, "Sul significato geometrico del secondo parametro differenziale di nLnia ftnnzione sopra una superficie qualunque," Rend. Lincei, ser. 5, 18, 309-316 (1909).

5. N. Kuznetsov, "Metaharmonic functions: mean flux theorem, its converse and related properties," Algebra \&5 Analiz, 33, no. 2, 82-97 (2021).

6. C. Neumann, Allgemeine Untersuchungen über das Newtonsche Prinzip der Fernwirkungen, Teubner, Leipzig (1896).

7. H. Poritsky, "Generalizations of the Gauss law of the spherical mean," Trans. Amer. Math. Soc. 43, 199-225 (1938).

8. F. John, Plane Waves and Spherical Means Applied to Partial Differential Equations, Interscience, New York (1955).

9. G. N. Watson, A Treatise on the Theory of Bessel Functions, 2nd ed., Cambridge University Press, Cambridge (1944).

10. A. P. Prudnikov, Yu. A. Brychkov and O. I. Marichev, Integrals and Series, vol. 2, Special Functions, Gordon \& Breach, New York et al. (1986).

11. S. G. Mikhlin, Mathematical Physics, an Advanced Course, North-Holland Publishing Co., Amsterdam-London (1970).

12. S. Axler, P. Bourdon and W. Ramey, Harmonic Function Theory, 2nd ed., Springer-Verlag, New York (2001).

13. V. S. Vladimirov, Equations of Mathematical Physics, Marcel Dekker, New York (1971). 
14. D. Gilbarg and N. S. Trudinger, Elliptic Partial Differential Equations of Second Order, Springer, Berlin etc. (1983).

15. O. D. Kellogg, Foundations of Potential Theory, Springer, Berlin (1929).

16. O. D. Kellogg, "Converses of Gauss' theorem on the arithmetic mean," Trans. Amer. Math. Soc. 36, 227-242 (1934).

17. J. R. Baxter, "Restricted mean values and harmonic functions," Trans. Amer. Math. Soc. 167, 451-463 (1972).

18. N. Kuznetsov, "Mean value properties of harmonic functions and related topics (a survey)," J. Math. Sciences, 242, 177-199 (2019).

19. N. Wiener, "The Dirichlet problem," J. Math. and Phys. 3, 127-147 (1924).

20. O. A. Oleinik, "On the Dirichlet problem for equations of elliptic type," Matem. Sbornik, 24, 3-14 (1949) (in Russian).

21. G. Tautz, "Zur Theorie der ersten Randwertaufgaben," Math. Nachr., 2, 279-303 (1949).

22. C. Miranda, Partial Differential Equations of Elliptic Type, 2nd ed., Springer, Berlin etc. (1970). 